\title{
Is segmental muscle strength recovery different in patients with dominant versus non-dominant hemispheric ischemic stroke?
} Dana Marieta Fodor ${ }^{1}$, Ingrid Mutter ${ }^{1}$, Ioana Cristina Stănescu ${ }^{1}$, Marius Fodor ${ }^{2}$, Angelo Bulboaca ${ }^{1}$,
Lăcrămioara Perju Dumbravă1

Corresponding author: Ingrid Mutter, E-mail: ingrid.mutter015@gmail.com,

${ }^{1}$ Neuroscience Department, Iuliu Haţieganu University of Medicine and Pharmacy, Cluj Napoca, Romania ${ }^{2}$ Vascular Surgery Department, Emergency County Hospital, Cluj-Napoca, Romania

\begin{abstract}
Introduction \& objectives: Starting from the well-known functional hemispheric asymmetry and concomitantly, from the clinical findings of practitioners involved in the post-stroke rehabilitation process, the aim of this study was to evaluate whether ischemic stroke in the dominant hemisphere results in more severe initial motor deficit and if its motor recovery is decreased compared to that of the non-dominant hemisphere.
\end{abstract}

Material and method: This was a retrospective study, comprising 39 patients with ischemic stroke in the middle cerebral artery territory, divided into two groups depending on the hemispheric location of the lesion (left/right). They were evaluated for their segmental muscle strength using the Medical Research Council Muscle Strength Grading Scale, both in terms of initial value and evolution between two successive admissions.

Results and conclusions: No significant difference was found for motor deficit evaluated on the occasion of the first admission between patients with left-side stroke and those with right-side stroke. Motor recovery was more obvious proximally in the paretic limbs, but without statistical significance.

Key words: hemispheric dominance, functional laterality, stroke, motor rehabilitation,

\section{Introduction}

Functional laterality correlated with hemispheric dominance is innate and genetically controlled, even if the genes involved are not yet well-known. Functional asymmetry between the two hemispheres is recognized, the left hemisphere being related to reason, logic and language, while the right hemisphere is associated with creativity and visualspatial perception. Ninety percent of individuals are right-handed (dominant left hemisphere) and the rest of about $10 \%$ are left-handed (dominant right hemisphere) (1-3).

Stroke in the middle cerebral artery (sylvian artery) territory will result in contralateral hemiparesis, possibly contralateral hemihypoesthesia and/or hemi/quadrantanopia, but other associated symptoms depend on the hemispheric location of the lesion. Left side location will be associated with aphasia or apraxia, while right side location will associate spatial neglect contralateral to the lesion, anosognosia, visual spatial perceptual disorder, body image deficit, postural malalignment. This explains the hypothesis that a left-side sylvian ischemic stroke will lead to more severe motor deficit, the left hemisphere playing a more important role in the control of motor behavior compared to the right hemisphere (4-6). On the other hand the right side stroke seems to be more common and also associated with significantly higher morbidity (7). Can the stroke hemispheric localization be considered as a biomarker beside the neuroimaging, electrophysiological or biochemical biomarkers? Moreover, can it contribute to the motor outcome prediction? (8-10)

\section{Material and method}

A retrospective study was conducted, which included 39 patients with ischemic stroke in the sylvian territory (middle cerebral artery - MCA) with contralateral hemiparesis, admitted to the services of Neurology I and Neurology II of the Rehabilitation Hospital Cluj-Napoca, in the period 01.01.201730.06 .2018 (1.5 years). The inclusion criteria were age over 18 years, stroke occurring within the last 2 
years, its confirmation by neuroimaging, and 2 consecutive admissions for motor rehabilitation in the mentioned time period. From the observation sheets, demographic data, the hemispheric location of the cerebral infarction and the Medical Research Council Muscle Strength Grading Scale (MRC S) values for paretic limbs (proximal and distal) were collected; for each admission, 4 values of MRC S were obtained which corresponded to upper limb-proximal (ULP), upper limb-distal (ULD), lower limb-proximal (LLP) and lower limb-distal (LLD). The patients were assigned to two groups according to the hemispheric location (right/left) of the stroke. Due to the retrospective lack of data related to the hemispheric dominance of the patients, it was considered that the left hemisphere was dominant and the right hemisphere was non-dominant in the studied patients (based on the literature statistical data, $90 \%$ of individuals being right-handed).

The Medical Research Council Muscle Strength Grading Scale (MRC S) is an assessment tool for segmental motor deficit including 5 grades: $0-$ no movement; 1 - flicker or trace contraction; 2 - active movement with gravity eliminated; 3 - active movements against gravity but not against resistance; 4 - active movements against resistance; 5 - normal power (11).

Statistical analysis was performed using Microsoft Excel. Categorical data were presented as diagrams, absolute and relative frequencies, and continuous variables were summarized using synthetic centrality, dispersion and location indicators or frequency histograms. Statistical analysis used the paired and unpaired $t$ test. A $p$ value lower than 0.05 allows rejection of the null hypothesis ( $\mathrm{H} 0)$ and acceptance of the alternative hypothesis (H1).

\section{Results}

Of the 39 patients, $36 \%$ were women and $64 \%$ were men, distributed by age groups as shown in Figure 1. Regarding the two groups of patients, with left MCA stroke and right MCA stroke, they were relatively equal both in terms of number of patients in each group and sex distribution (Table 1, Fig 1).
Table 1: Distribution of the two hemispheric locations depending on gender

\begin{tabular}{|l|l|l|}
\hline Sex & Left MCA stroke & Right MCA stroke \\
\hline Women & 7 & 7 \\
\hline Men & 12 & 13 \\
\hline Total & 19 & 20 \\
\hline
\end{tabular}

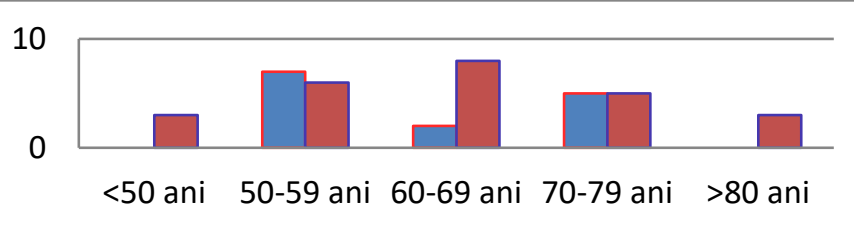

Fig. 1. Distribution of cases by age and sex (blue - women, red - men) By comparing the mean MRC scale (MRC S) values for the paretic limbs (ULP, ULD, LLP, LLD) using the paired $\mathbf{t}$ test, statistically significant increases (improvements) were found for both paretic limbs, both proximally and distally $(\mathrm{p}<0.05)$, especially for the proximal upper limb (Fig.2).

By comparing the initial mean MRC $S$ values between patients with left MCA stroke and right MCA stroke (reflecting the initial motor deficit at the patients' presentation to the Rehabilitation Hospital), no statistically significant results were obtained; so, the null hypothesis that the motor deficit assessed by MRC S is similar in the case of left-side stroke (dominant hemisphere) and right-side stroke (nondominant hemisphere) is not rejected, but we can observe a lower mean value of MCR for the proximal region of the paretic upper limb (Fig. 3).

The two groups (right MCA stroke and left MCA stroke) were also compared for the difference of the mean values by which MRC S improved between the two admissions (Fig. 4) using the $t$ unpaired test (Fig. 4). It can be seen that the improvement in MRC S values for the proximal region of both the upper limb and the lower limb is more obvious in the case of the right side location of stroke, while for the distal region of the upper limb the improvement is more marked for the right side location of stroke and is about the same for the distal region of the lower limb. However, $p$ values $>0.05$ do not allow to reject the null hypothesis that there is no significant difference in the evolution of motor deficit in paretic limbs between the left and right side location of ischemic stroke. 


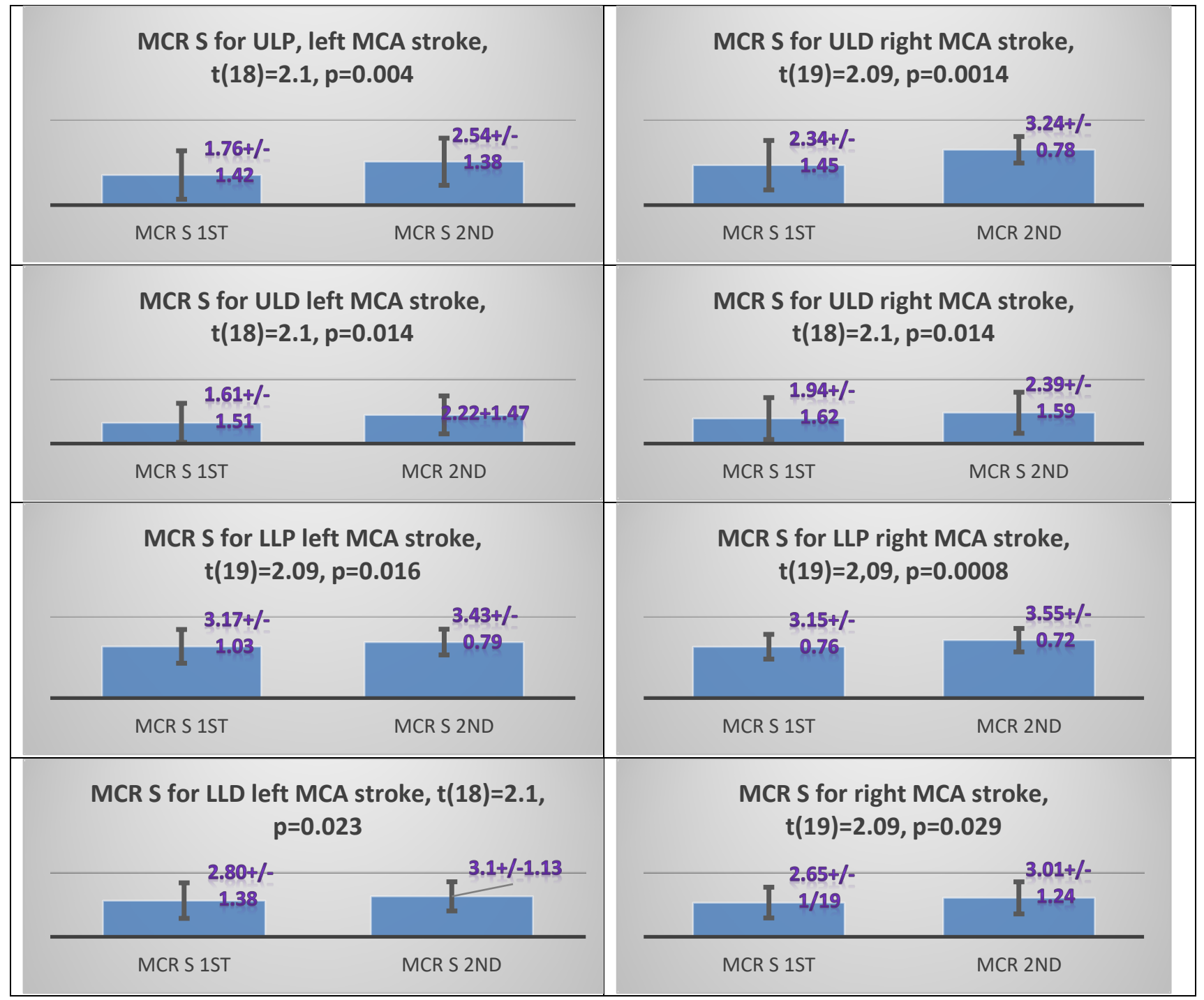

Fig.2. The improvement of MCR S for each paretic limb (proximal and distal) for left and right MCA stroke, according to paired $\mathbf{t}$ test analysis.

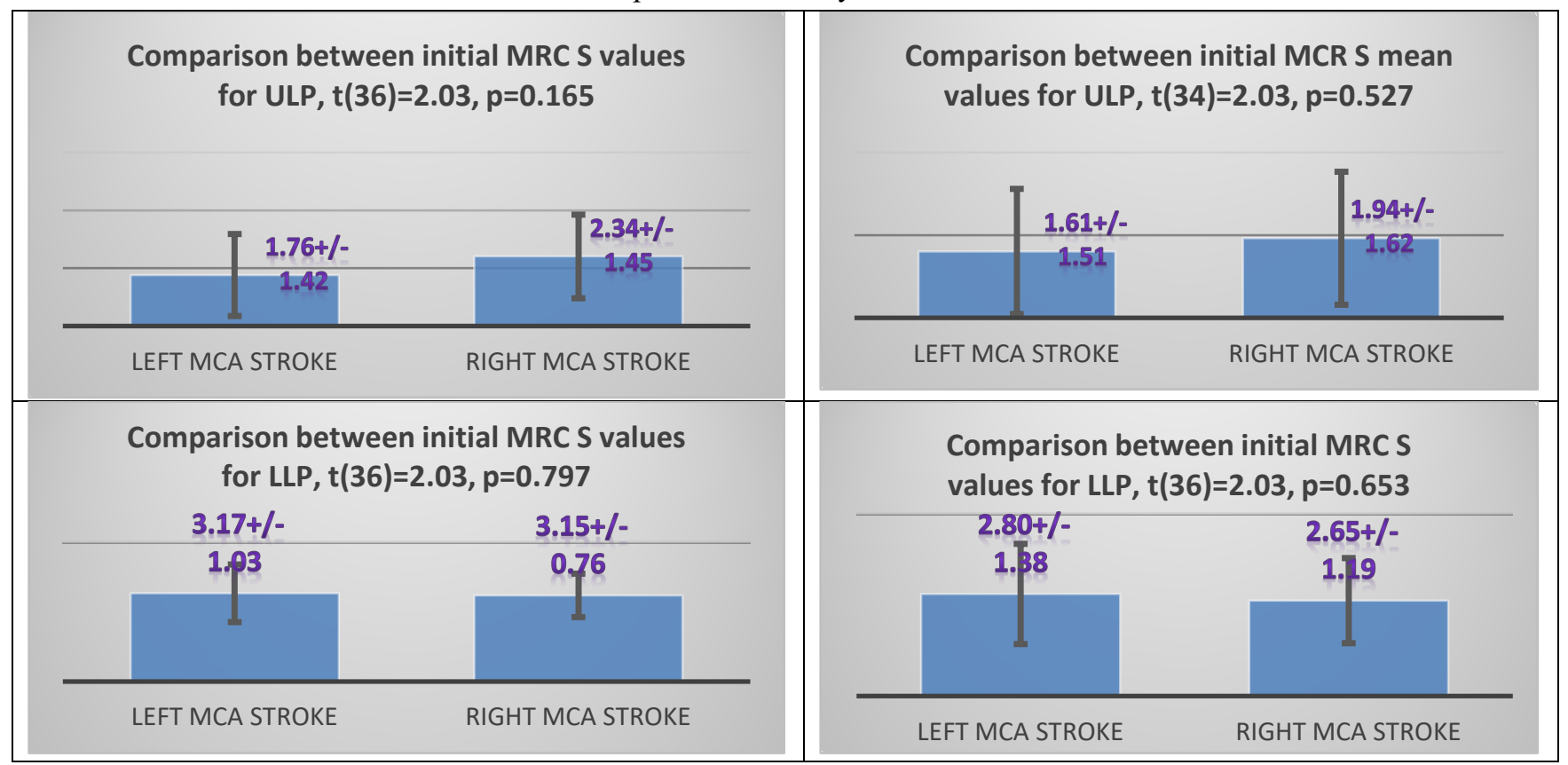

Fig. 3. Initial mean values for MRC S (left vs right MCA stroke) according to unpaired $\mathbf{t}$ test analysis (first admission) 


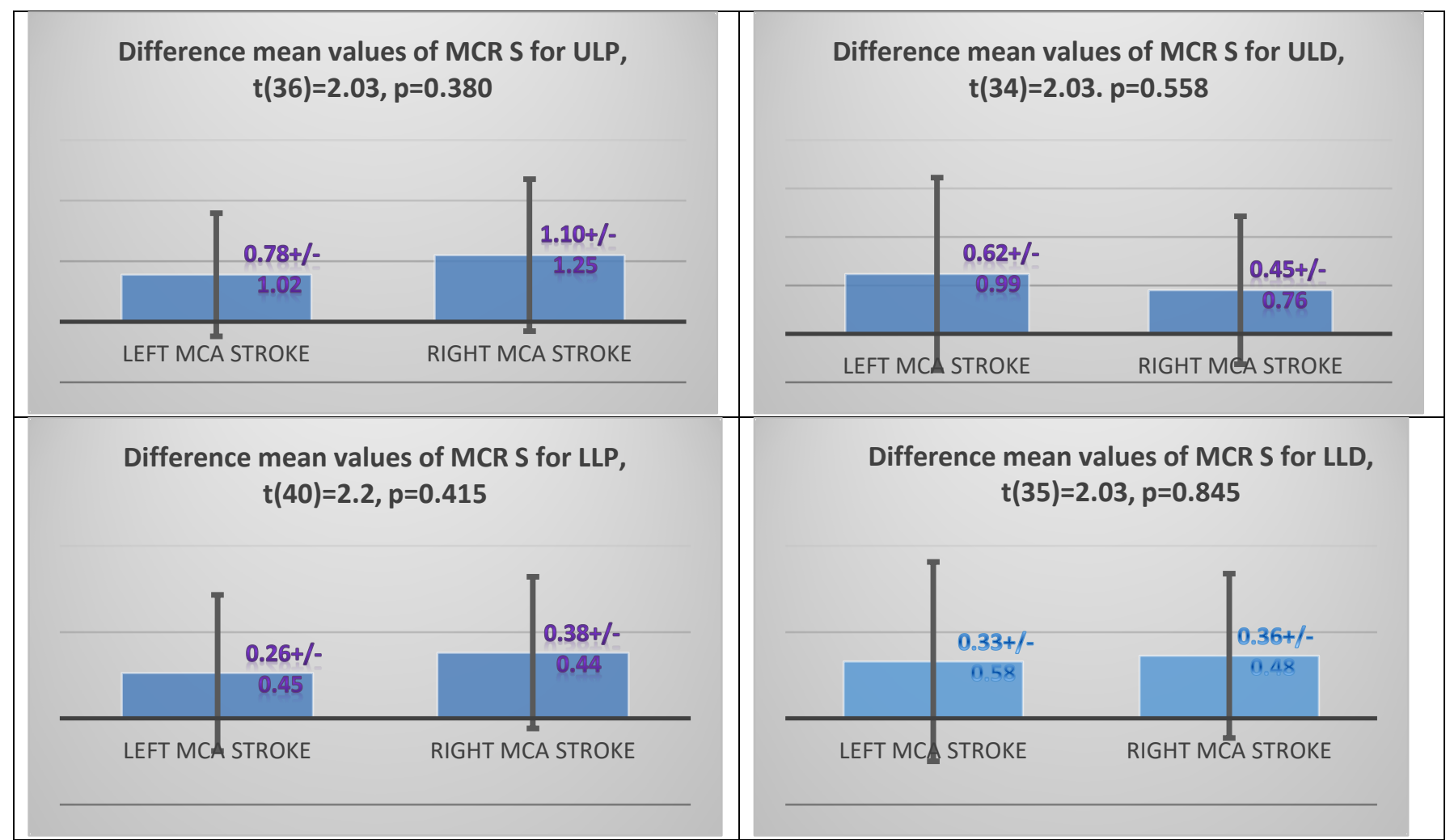

Fig.4. Difference MRC S mean values for each limb (proximal and distal), left vs right MCA stroke, between the two admissions, according to unpaired $\mathbf{t}$ test analysis

\section{Conclusion}

In healthy persons, the dominant upper limb (the right limb for the majority) is superior to the non-dominant one in tasks that demand precision, speed, coordination and muscle strength. Consequently, clinical practitioners (neurologists, kinesiotherapists, occupational therapists) frequently believe that motor deficit is more pronounced in the case of left-side stroke and functional rehabilitation of patients with left-side stroke is worse than that of patients with right-side stroke, supposing that impairments of the dominant (right) upper limb would be more detrimental for activities of daily living compared to impairments of the non-dominant side (left), supported by some reports $(4,5)$ and not by others $(12,13)$. Our research could not validate this hypothesis for the grade of motor deficit (measured by the MRC Muscle Strength Grading Scale at the first admission).

Also, for the degree of motor deficit recovery (reflected by the difference in MRC Muscle Strength Grading Scale between the second and the first

admission), the results were not statistically significant, but a greater improvement in proximal muscle strength in the paretic limbs was observed in our study for the right side location. Due to the retrospective nature of the study, only the data available in the observation sheets were used, while information about the assessment of the evolution of other aspects of motor rehabilitation such as coordination, posture and walking was absent. Associating this aspects some authors concluded that left MCA strokes have a worse outcome than their right-hemispheric counterparts $(4,5)$. More extensive studies are probably required, which can provide additional information based on a differentiated approach to patients with dominant versus nondominant hemispheric stroke. 


\section{References}

1. Corballis MC. Left brain, right brain: facts and fantasies. PLoS biology. 2014;12(1):e1001767.

2. Armour JA, Davison A, McManus IC. Genomewide association study of handedness excludes simple genetic models. Heredity. 2014;112(3):221-5.

3. McManus IC, Davison A, Armour JA. Multilocus genetic models of handedness closely resemble single-locus models in explaining family data and are compatible with genomewide association studies. Annals of the New York Academy of Sciences. 2013;1288:48-58.

4. Hedna VS, Bodhit AN, Ansari S, Falchook AD, Stead L, Heilman KM, et al. Hemispheric differences in ischemic stroke: is left-hemisphere stroke more common? J Clin Neurol. 2013;9(2):97-102.

5. Voos MC RdVL. Comparative study on the relationship between stroke hemisphere and functional evolution in right-handed individuals. Rev bras fisioter. 2008 [cited 2019 Apr 16] 12(2):113-20.

6. Stanescu I, Dogaru G. Cognitive rehabilitation: an important tool in disability improvement after brain injuries. Balneo Research Journal. 2016;7(3):89-96.

7. Rastogi V, Lamb DG, Williamson JB, Stead TS, Penumudi R, Bidari S, et al. Hemispheric differences in malignant middle cerebral artery stroke. Journal of the neurological sciences. 2015;353(1-2):20-7.
8. Laredo C, Zhao Y, Rudilosso S, Renu A, Pariente JC, Chamorro A, et al. Prognostic Significance of Infarct Size and Location: The Case of Insular Stroke. Scientific reports. 2018;8(1):9498.

9. Hayward KS, Schmidt J, Lohse KR, Peters S, Bernhardt J, Lannin NA, et al. Are we armed with the right data? Pooled individual data review of biomarkers in people with severe upper limb impairment after stroke. NeuroImage Clinical. 2017;13:310-9.

10. Stanescu I, Dogaru G. Brain Natriuretic Peptide (Bnp): Biomarker for Risk Stratification and Functional Recovery Prediction in Ischemic Stroke. Balneo Research Journal. 2015;6(1):404.

11. James MA. Use of the Medical Research Council muscle strength grading system in the upper extremity. The Journal of hand surgery. 2007;32(2):154-6.

12. Fink JN, Frampton CM, Lyden P, Lees KR. Does hemispheric lateralization influence functional and cardiovascular outcomes after stroke?: an analysis of placebo-treated patients from prospective acute stroke trials. Stroke. 2008;39(12):3335-40.

13. McCombe Waller S, Whitall J. Hand dominance and side of stroke affect rehabilitation in chronic stroke. Clinical rehabilitation. 2005;19(5):54451 . 